\title{
Interactions Between Avidin and Graphene for Development of a Biosensing Platform
}

Isaac Macwan

Fairfield University, imacwan@fairfield.edu

Md Daud Hossain Khan

Ashish Aphale

Shrishti Singh

P\&lpoWLiłis and additional works at: https://digitalcommons.fairfield.edu/engineering-facultypubs (C) 2016 Elsevier B.V. All rights reserved.

She author past-printibas, been archived here with permission from the copyright holder under a CC-BY-NC-ND license.

\section{Peer Reviewed}

\section{Repository Citation}

Macwan, Isaac; Khan, Md Daud Hossain; Aphale, Ashish; Singh, Shrishti; Liu, Juan; Higorani, Manju; and Patra, Prabir K., "Interactions Between Avidin and Graphene for Development of a Biosensing Platform" (2017). Engineering Faculty Publications. 304.

https://digitalcommons.fairfield.edu/engineering-facultypubs/304

\section{Published Citation}

Macwan, Isaac, Md Daud Hossain Khan, Ashish Aphale, Shrishti Singh, Juan Liu, Manju Hingorani, and Prabir Patra. “Interactions Between Avidin and Graphene for Development of a Biosensing Platform." Biosensors \& Bioelectronics. 89 (March 2017): 326-33. https://doi.org/10.1016/j.bios.2016.07.024.

This item has been accepted for inclusion in DigitalCommons@Fairfield by an authorized administrator of DigitalCommons@Fairfield. It is brought to you by DigitalCommons@Fairfield with permission from the rightsholder(s) and is protected by copyright and/or related rights. You are free to use this item in any way that is permitted by the copyright and related rights legislation that applies to your use. For other uses, you need to obtain permission from the rights-holder(s) directly, unless additional rights are indicated by a Creative Commons license in the record and/or on the work itself. For more information, please contact digitalcommons@fairfield.edu. 


\section{Authors}

Isaac Macwan, Md Daud Hossain Khan, Ashish Aphale, Shrishti Singh, Juan Liu, Manju Higorani, and Prabir K. Patra 
and Bioelectronics

Manuscript Number: BIOS-D-16-00410

Title: INTERACTIONS BETWEEN AVIDIN AND GRAPHENE FOR DEVELOPMENT OF A BIOSENSING PLATEORM

Article Type: SI:2D Materials

Section/Category: The others

Keywords: Graphene, Avidin, Biosensors, Molecular Dynamics, Electrochemical Impedance

Corresponding Author: Dr. Prabir Patra, PhD

Corresponding Author's Institution: University of Bridgeport

First Author: Isaac Macwan, PhD

Order of Authors: Isaac Macwan, PhD; Mohammad Daud Khan, MS; Ashish Aphale, PhD; Shrishti Singh, MS; Juan Liu, PhD; Manju Hingorani, PhD; Prabir Patra, PhD 


\title{
INTERACTIONS BETWEEN AVIDIN AND GRAPHENE FOR DEVELOPMENT OF A BIOSENSING PLATFORM
}

Isaac Macwan*a,b ${ }^{\text {a, }}$ Md. Daud Hossain Khan ${ }^{\mathrm{b}}$, Ashish Aphale ${ }^{\mathrm{b}}$, Shrishti Singh ${ }^{\mathrm{b}}$, Juan Liu ${ }^{\mathrm{c}}$, Manju M. Hingorani ${ }^{c}$, Prabir Patra*b,d

${ }^{a}$ Department of Computer Engineering, University of Bridgeport, Bridgeport CT 06604 USA

${ }^{\mathrm{b}}$ Department of Biomedical Engineering, University of Bridgeport CT 06604 USA

${ }^{c}$ Department of Molecular Biology and Biochemistry, Wesleyan University, Middletown CT 06459 USA

${ }^{\mathrm{d}}$ Department of Mechanical Engineering, University of Bridgeport, Bridgeport CT 06604 USA

Email Addresses:

Isaac Macwan (*Corresponding Author): - imacwan@bridgeport.edu

126 Park Avenue, Bridgeport CT 06604

(203)385-2129

Md. Daud Hossain Khan: - mdkhan@ my.bridgeport.edu

Ashish Aphale: - aaphale@ bridgeport.edu

Shrishti Singh: - shrsingh@my.bridgeport.edu

Juan Liu: - jliu03@wesleyan.edu

Manju Hingorani: - mhingorani@wesleyan.edu

Prabir Patra (*Corresponding Author): - ppatra@ bridgeport.edu

126 Park Avenue, Bridgeport CT 06604

(203)576-4165

\begin{abstract}
Fundamental understanding of interactions at the interface of biological molecules, such as proteins, and nanomaterials is crucial for developing various biocompatible hybrid materials and biosensing platforms. Conductive nanomaterials-based biosensors offer the advantage of higher sensitivity and reliable diagnosis mainly due to their superior specific surface area and ballistic conductivity. Conductive nanocomposite films that immobilize proteins can synergize the properties of transducers and molecular recognition elements in order to improve biosensor performance and diversity. Here we report for the first time, using a combined molecular dynamics simulations and experimental approach, the interactions between avidin and a graphene surface, which is being developed as a sensing platform for early detection of DNA mismatch repair proteins. We find that the interactive forces between avidin and graphene are mainly hydrophobic, along with some van der Waals, electrostatic and hydrogen bonding interactions. Notably, the structure and function of the avidin molecule is preserved after its adsorption on the graphene surface. The MD results agree well with scanning electron microscopy (SEM) and electrochemical impedance spectroscopy (EIS) analysis of avidin immobilized on a graphenated polypyrrole (G-PPy) conductive substrate, which confirm adsorption of avidin on graphene nanoplatelets and corresponding changes in electrical impedance, respectively.
\end{abstract}


KEYWORDS: Graphene, Avidin, Biosensors, Molecular Dynamics, Electrochemical Impedance

\section{Introduction}

With the advance of nanobiotechnology, the investigation of biological macromolecules and nanomaterials has progressed to molecular and atomic levels with a focus on understanding and controlling the interfaces between them (Sarikaya et al., 2003; Zhao et al., 2008; Nel et al., 2009; Kostarelos and Novoselov, 2014). Research on the interface between protein and conducting nanomaterials, such as graphene and carbon nanotubes, has revolutionized industries particularly interested in developing hybrid biomaterials and biosensors (Zhang et al., 2013; Georgakilas et al., 2012; Hu et al., 2011; Lu et al., 2009; Gräslund et al., 2008; Xue et al., 2014).

Biosensors are now ubiquitous in medical diagnostics and analysis systems in order to detect and quantify macromolecules such as oligonucleotides and proteins. One of the primary performance matrices of a biosensor is its sensitivity, which depends on the design and synthesis of the interface between the biological recognition element and the transducer. Graphene, due to its superior mechanical and electrical properties, has been the center of interest in the development of biosensors (Rodrigo et al., 2015; Zhang et al., 2015; Kuila et al., 2011; Shao et al., 2010). Advances in DNA immobilization techniques also point to the use of conductive polymers such as electropolymerized polypyrrole in avidin/biotin systems as a means of irreversibly immobilizing oligonucleotides to form DNA-based biosensors (Dupont-Filliard et al., 2001; Ouerghi et al., 2002;(Dupont-Filliard et al., 2004). These methodologies typically utilize impedance measurement for label-free detection of target molecules (Daniels and Pourmand, 2007; Drummond et al., 2003; Sadik et al., 2009). Combining these concepts, the development of a biotinylated-DNA-avidin-graphene based biosensor is a plausible approach for functional detection of DNA-binding protein biomarkers (for example, mutant DNA mismatch repair proteins associated with Lynch Syndrome (Tiwari et al., 2015)).

Development of such sensitive biosensors demands a thorough understanding of the interactions at the interface of single-layer graphene and avidin molecules. It has been previously reported that protein adsorption on a graphene surface is minimal, mainly due to hydrophobic interactions (Russo and Passmore, 2014). It is also known that chemical modifications or the presence of support substrates can substantively improve the adsorption of biomolecules on the surface of graphene/graphene oxide (Lee et al., 2011; Yokota et al., 2011; Alava et al., 2013). More recently, it has been shown that avidin can selectively deposit on graphene flakes in the presence of a chemically modified $\mathrm{SiO}_{2} / \mathrm{Si}$ support substrate (Kamiya et al., 2014). However, the process of self-assembly between a graphene surface and avidin molecule through strong nonbonding interactions is not understood in detail. This knowledge is particularly important in the case of proteins because their rapidly changing conformations can alter the interactions within a matter of few femtoseconds, making physical quantification extremely difficult. Molecular Dynamics (MD) simulations can be used effectively to address this problem. Previous MD simulation data on avidin are mostly concerned with the formation or dissociation of the avidinbiotin complex and the energy changes involved therein (Wilchek and Bayer, 1988; Evans and Ritchie, 1997; Izrailev et al., 1997; Isralewitz et al., 2001); however, to our knowledge there are no such data available on interactions between avidin and other macromolecules, especially nanoparticles. In contrast, there is a plethora of MD simulations on graphene alone to determine its various physical properties such as thermal and electrical conductivity as well as mechanical properties such as stress, strain and Young's modulus (Hu et al., 2009; Jiang et al., 2009; Pei et al., 2010; Zhong et al., 2011). However, even in this case, only limited data are available on 
integrated atomic MD simulations of graphene and avidin. For instance, Haddad and colleagues have reported the only MD simulation study of the interactions of avidin and single-walled carbon nanotubes wherein avidin was deposited on biotinylated carbon nanotubes, the latter being assembled via pyrrole electro-polymerization (Haddad et al., 2009). Hence, a survey of recent literature indicates that limited information on direct interactions between graphene and avidin is currently available.

Given that additional details on the interface between avidin and nanomaterials could significantly improve the design of biosensing platforms, we conducted a MD simulation study of the adsorption of avidin on the surface of non-functionalized graphene. We found that the interaction forces between avidin and graphene are mainly hydrophobic, along with some van der Waals, electrostatic, and hydrogen bonding interactions. Importantly, the structure and function of avidin is preserved even after its adsorption on the graphene surface. Furthermore, the results from the MD simulations are supported by scanning electron microscopy (SEM) and electrochemical impedance spectroscopy (EIS) experiments examining the morphological features of avidin immobilized on a graphenated polypyrrole (G-PPy) conductive substrate. The findings offer new insights into graphene-avidin interactions that can be applied towards a novel 'DNA-avidin-G-PPy' biosensing platform for detection of DNA binding proteins. 


\section{Materials and Methods}

\subsection{Molecular dynamics simulations and analysis of the interactions between avidin and}

graphene

Interactive forces between graphene and avidin were set up and analyzed using Visual MD (VMD) (Humphrey et al., 1996) and the simulations were carried out using NAMD (Phillips et al., 2005). Note that since avidin can selectively deposit on exposed graphene flakes in the presence of other substrates as reported by Kamiya and colleagues (Kamiya et al., 2014), pyrrole was excluded from the simulations. The avidin pdb file (2AVI) was obtained from the protein data bank and an inbuilt graphene sheet builder plugin in the VMD was used to create a single layer graphene sheet of dimensions $88 \AA$ x $121.44 \AA$. All-atom simulations containing a singlebarrel avidin monomer (120 residues) and a single layer graphene sheet (4,292 atoms) were carried out for a time period of $100 \mathrm{~ns}$. All simulations used the CHARMM (MacKerell, et al., 1998) force field and TIP3 (Jorgensen et al., 1983) water model with a neutralizing salt concentration of $\mathrm{NaCl}$ for effective polarization of the water molecules. A cluster of four $\mathrm{HP}$ Z230 systems with a total of 32 cores each using Intel Xeon processor and Quadro K620 CUDA acceleration capability were used to perform all simulations.

In each simulation, the temperature was maintained at $300 \mathrm{~K}$ by Langevin thermostat and a pressure of $1 \mathrm{~atm}$ through Nose-Hoover Langevin-Piston barostat with a period of $100 \mathrm{ps}$ and a decay rate of $50 \mathrm{ps}$ assuming the periodic boundary conditions. A 10,000-step energy minimization of the entire system (containing graphene, avidin, water, and $\mathrm{NaCl}$ ions) was performed first to reach a stable state. All atom-simulations employed an integrated time step of 2 fs. A cut-off of 10 to $12 \AA$ designated the short-range forces while long-range forces were calculated using Particle Mesh Ewald (PME) algorithm. Root Mean Square Deviation (RMSD) and NAMD energy extensions were used to determine interaction energy between avidin and graphene. VMD Timeline tool was used to determine the secondary structure of avidin and Root Mean Square Fluctuations (RMSF) as well as RMSD for the adsorbed residues. TCL scripting was used to determine the number of atoms per cut off distance from the graphene surface and interaction energy per number of atoms.

\subsection{Reagents and apparatus}

The chromium-plated nickel chips were purchased from ExpressPCB (Santa Monica, CA, USA), each consisting of a 6 finger, incomplete circuit embedded on a $4 \mathrm{~cm}$ x $2 \mathrm{~cm}$ plastic frame. Avidin (Egg white, unconjugated, $10 \mathrm{mg}$ ) and HEPES (1 M, $100 \mathrm{ml}, \mathrm{pH}$ 7.2-7.5) were obtained from Thermo Fisher Scientific (Waltham, MA, USA). Pyrrole (100 ml, reagent grade $98 \%$, vapor density 2.31 , density $0.967 \mathrm{~g} / \mathrm{ml}$ at $298 \mathrm{~K})$ and sodium sulfate $\left(\mathrm{Na}_{2} \mathrm{SO}_{4}\right.$, anhydrous, reagent grade, $500 \mathrm{~g}$ ) were purchased from Sigma-Aldrich (St. Louis, MO, USA) and Carolina Biological Supply (Burlington, NC, USA) respectively.

Electrochemical polymerization was performed using a conventional 3-electrode cyclic voltammetry setup (eDaq USA, Model number ER466): a working electrode (the chip) onto which the G-PPy substrate is formed, a platinum wire as the counter electrode (CE) and $\mathrm{Ag} / \mathrm{AgCl}$ (saturated $\mathrm{KCl}$ ) as the reference electrode (RE). EIS was performed using an electrochemical impedance analyzer (eDaq USA, ERZ 100). The morphology of the surface was analyzed using SEM (SEM, S-4800, Hitachi, Japan) with an acceleration voltage of $20 \mathrm{kV}$. Exposure time was fairly limited due to the denaturation of avidin under prolonged radiation. 


\subsection{Preparation of graphene-polypyrrole solution and its in-situ polymerization}

A modification of the method described previously by Aphale and colleagues was used for preparation of the G-PPy nanocomposite film (Aphale et al., 2015). The initial pyrrole-graphene homogenized mixture was prepared by dissolving $0.2 \mathrm{ml}$ of pyrrole, $0.355 \mathrm{~g}$ of sodium sulfate $\left(\mathrm{Na}_{2} \mathrm{SO}_{4}\right)$ and $0.01 \%(\mathrm{w} / \mathrm{v})$ in $25 \mathrm{ml}$ of demineralized water, followed by 30 minutes of stirring using a magnetic stirrer and 20 minutes of sonication in a cold water bath. The G-PPy film was then formed on the surface of the chip using potentiostatic electrochemical polymerization. The lower and upper voltage limits were $800 \mathrm{~V}$ and $900 \mathrm{~V}$ respectively, with a $20 \mathrm{mV} / \mathrm{s}$ scan rate. Formation of the G-PPy substrate was complete after 100 cycles. For morphology comparison, the developed G-PPy nanocomposite substrate was compared to a control substrate made by conventional or established procedures (Aphale, Maisuria et al. 2015).

\subsection{Preparation and deposition of avidin}

A modification of the method described previously by Kamiya and colleagues was used (Kamiya et al., 2014). Avidin was dispersed in 0.01 M N-2-hydroxyethylpiperazine-N'-2-ethanesulfonic acid solution (HEPES, pH 7.0) and G-PPy chips were soaked in the avidin solution for 45 minutes at room temperature $(300 \mathrm{~K})$. A highly concentrated avidin solution of $1 \mathrm{mg} / \mathrm{ml}$ was used in order to ensure complete engulfment of the protein on the exposed graphene flakes. Both experimental and control G-PPy films were soaked in avidin solution.

\subsection{Electrochemical impedance spectroscopy analysis}

EIS or impedance data was acquired during each stage of fabrication, yielding 3 graphical components; the impedance data of the bare Cr-Ni surface, G-PPy nanocomposite, and G-PPyavidin complex respectively. The EIS was only conducted after confirming the visual deposition of avidin onto the G-PPy nanocomposite substrate. A frequency range of $10 \mathrm{kHz}$ to $1 \mathrm{~Hz}$ and operating voltage of $100 \mathrm{mV}$ was used for the measurements. 


\section{Results}

\subsection{Simulation}

Figure 1 shows the interactions between a single layer graphene sheet and avidin monomer, with structures at the beginning of the MD simulation (Fig. 1A) and at $100 \mathrm{~ns}$ (Fig. 1B). The residues participating in the adsorption interface are shown using van der Waals (VDW) representation in VMD.

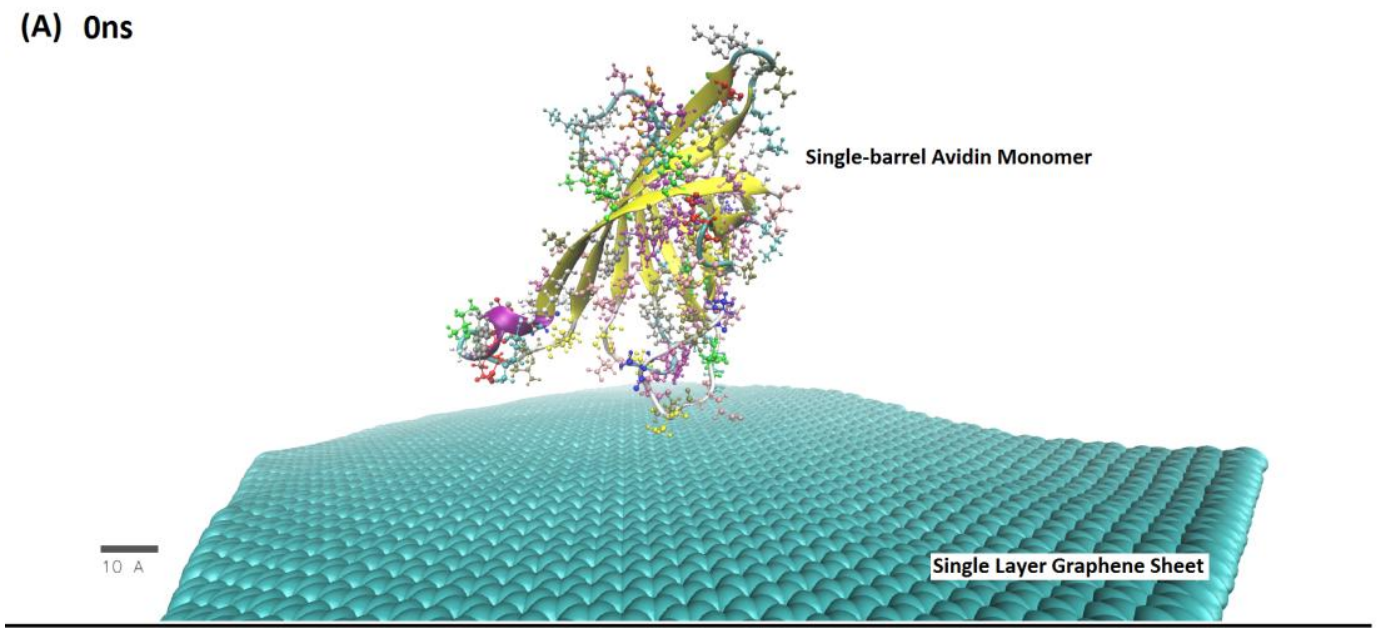

(B) $100 \mathrm{~ns}$

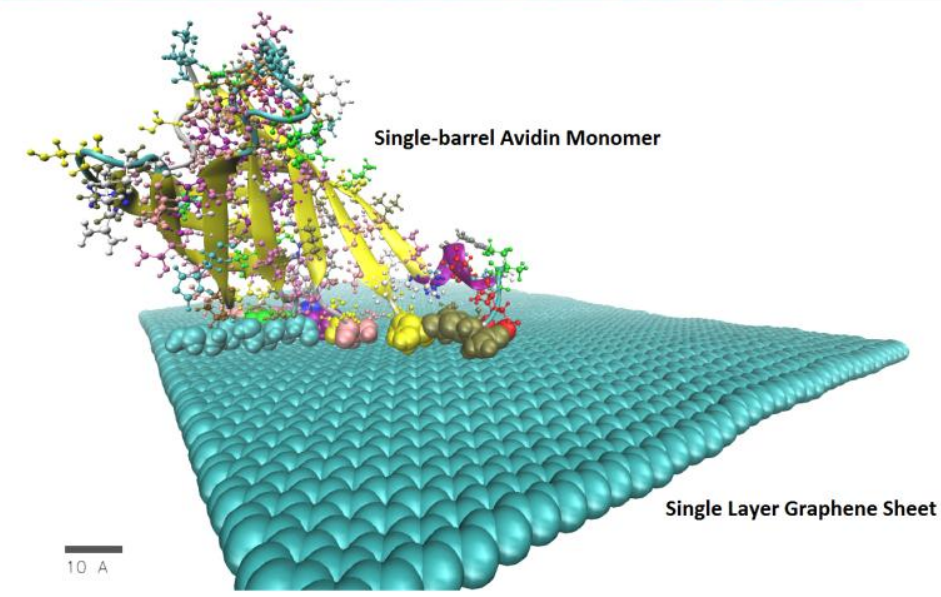

Fig. 1: Ball/stick representation of a single-barrel avidin monomer with a pristine single layer graphene sheet before (A) and after (B) a simulation run of 100ns (Total atoms: 78913). Water and neutralizing $\mathrm{NaCl}$ molecules are not shown for clarity.

In order to analyze the efficacy of avidin adsorption on the surface of a single layer graphene sheet, the distance between the center of mass of avidin and the graphene sheet was calculated from the VMD trajectory data, as shown in Figure 2A. To investigate the behavior of avidin monomer in the vicinity of the graphene sheet and the dominant forms of interactive energies at the interface between the two, non-bonding interactive forces including van der Waals and electrostatic interactions were plotted as a function of time $(100 \mathrm{~ns}=2500$ frames $)$, as shown in Figure 2B. 

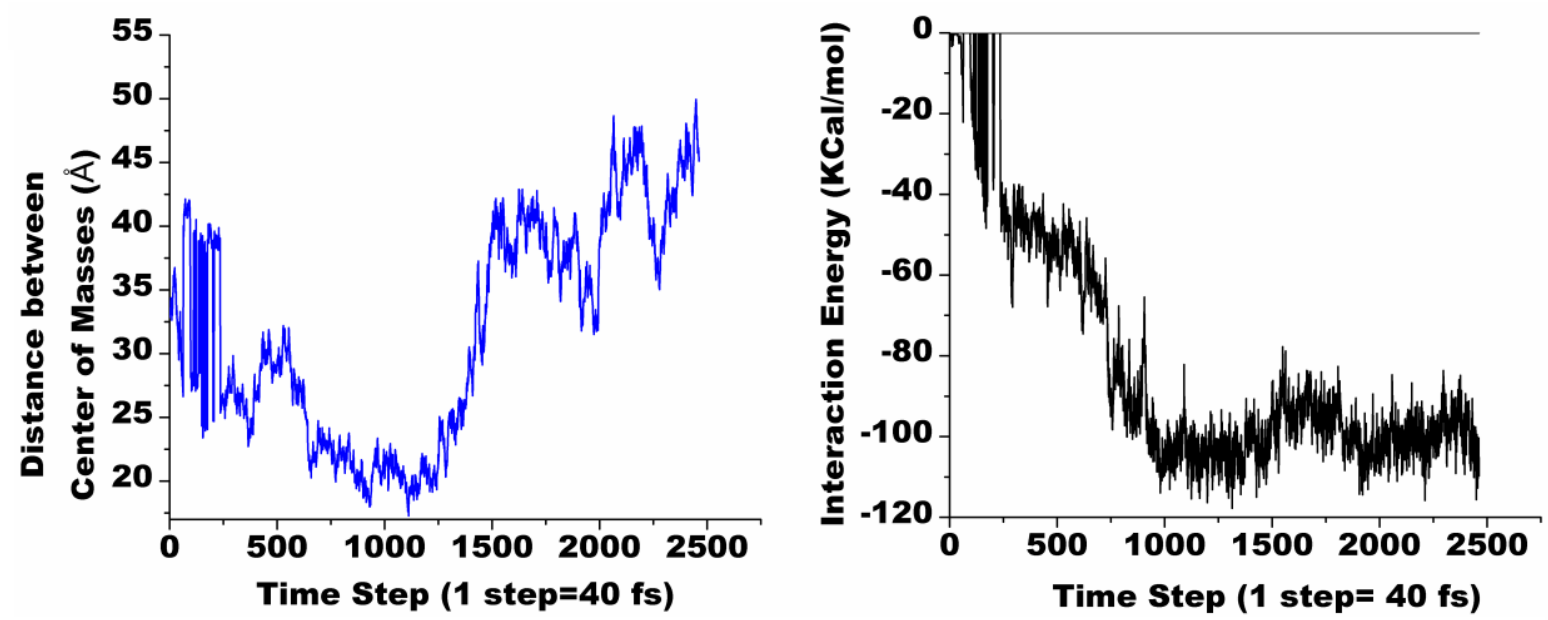

Fig. 2: (A) Changes in the distance between the center of mass of avidin and graphene sheet as a function of time; (B) Interaction Energy (VDW + Electrostatic) between avidin and graphene sheet as a function of time.

The avidin residues participating in adsorption on the graphene sheet are listed in Table 1. To quantify the adsorption process through these residues, a detailed RMSD analysis for the entire protein and the individual adsorbed residues was also performed using the RMSD trajectory and timeline tools in VMD. The results are as shown in Figures 3A and 3B.

\begin{tabular}{|l|l|l|}
\hline Residue Name & Residue ID & Type of Residue \\
\hline Threonine & 40 & Polar, un-charged \\
\hline Serine & $41,73,101,102,75$ & Polar, un-charged \\
\hline Asparagine & 42,104 & Polar, un-charged \\
\hline Glutamic Acid & 43,74 & Acidic, negatively charged side chain \\
\hline Isoleucine & 44 & Hydrophobic side chain \\
\hline Alanine & 39 & Hydrophobic side chain \\
\hline Lysine & $71,111,45$ & Basic, polar, positively charged chain \\
\hline Phenylalanine & 72 & Hydrophobic side chain \\
\hline Valine & 103 & Hydrophobic side chain \\
\hline Aspartic Acid & 105,108 & Acidic, negatively charged side chain \\
\hline
\end{tabular}

TABLE 1: Residues of avidin within $5 \AA$ of the graphene surface (criteria for adsorption in the present study, (Gu et al., 2015))

To determine the optimum distance from the graphene surface where different intermolecular forces are dominant, the atoms of all the adsorbed residues, including and excluding hydrogen, were analyzed. Based on these data, the cut-off distance from the graphene surface was plotted in terms of the number of adsorbing atoms, total interaction energy, and the interaction energy per atom of the adsorbing residue, as shown in Figures 3C, 3D and 3E. 

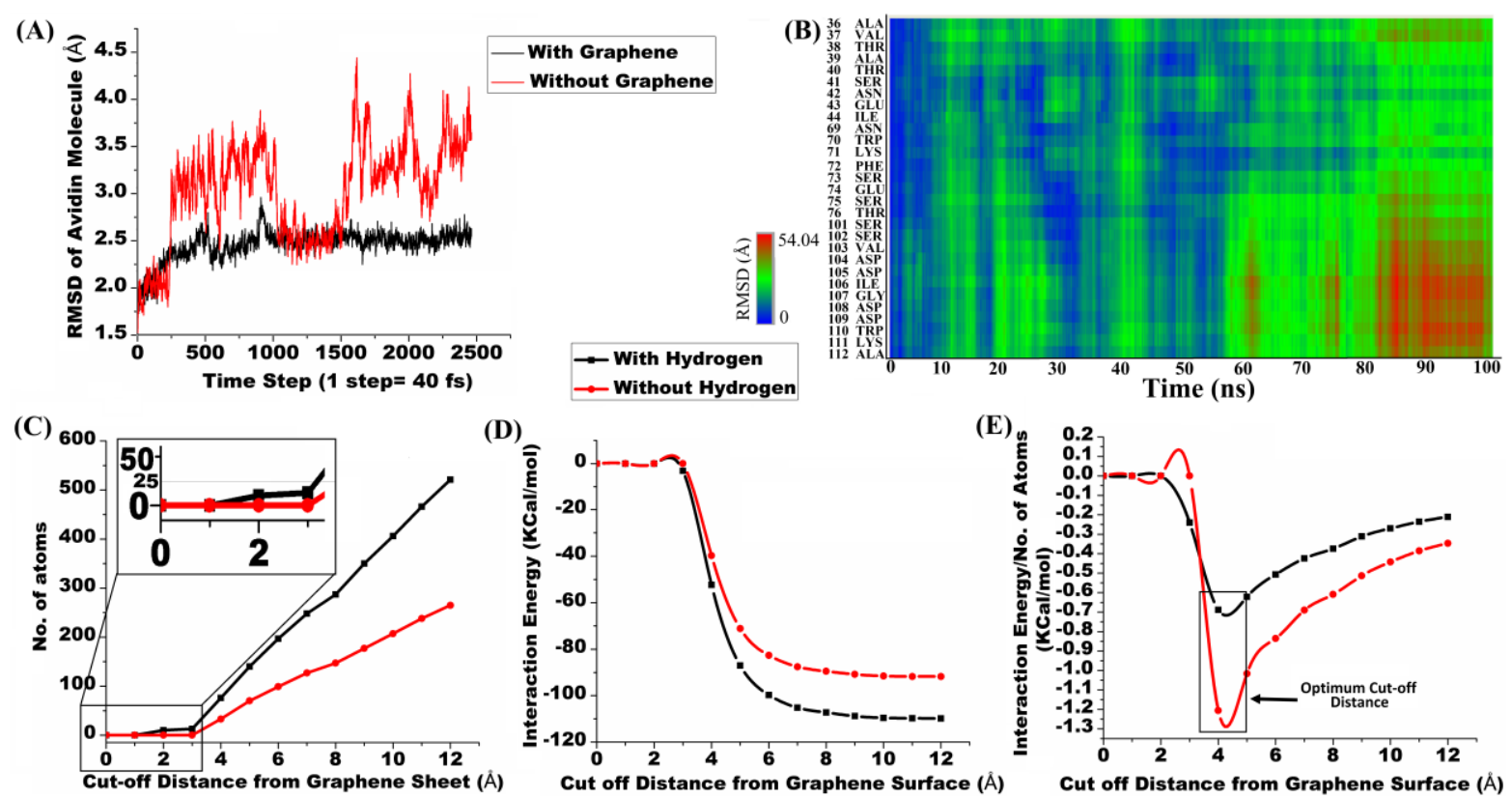

Fig. 3: (A) RMSD analysis of avidin showing stable adsorption over time on the surface of graphene sheet after 50ns (1250 time steps); (B) RMSD analysis of individual avidin residues illustrating the most dominant residues in adsorption in accordance with Table 1; (C) Plot showing the number of atoms of avidin as a function of cut-off distance from the surface of graphene including and excluding hydrogen. Inset: Blown-up view showing the dominance of hydrogen bonding below $3 \AA$. (D) Interaction energy as a function of cut-off distance from the surface of graphene with and without hydrogen atoms. (E) The ratio of interaction energy/number of atoms as a function of cut-off distance from the graphene sheet with and without hydrogen.

To understand the interaction between hydrogen atoms and the graphene sheet, especially between the distances of $1 \AA$ and $3 \AA$, which is commonly used to define hydrogen bonds (Jeffrey, 1997), the strength and life times of hydrogen bonds between the avidin residues and graphene were analyzed, as shown in Figure 4A. The electrostatic interaction energy between avidin and graphene was also monitored, as shown in Figure 4B. Moreover, since the ability of avidin to bind a biotinylated entity is of prime importance in any biosensing device, the stability of the secondary structure of avidin during the adsorption was calculated through the RMSD of the $\beta$ sheets and the 3-10-helix as shown in Figure 4C. Similarly, the stability of the loops L3, L5 and L7 was also monitored as they were being adsorbed onto the graphene sheet, as shown in Figure 4D. 

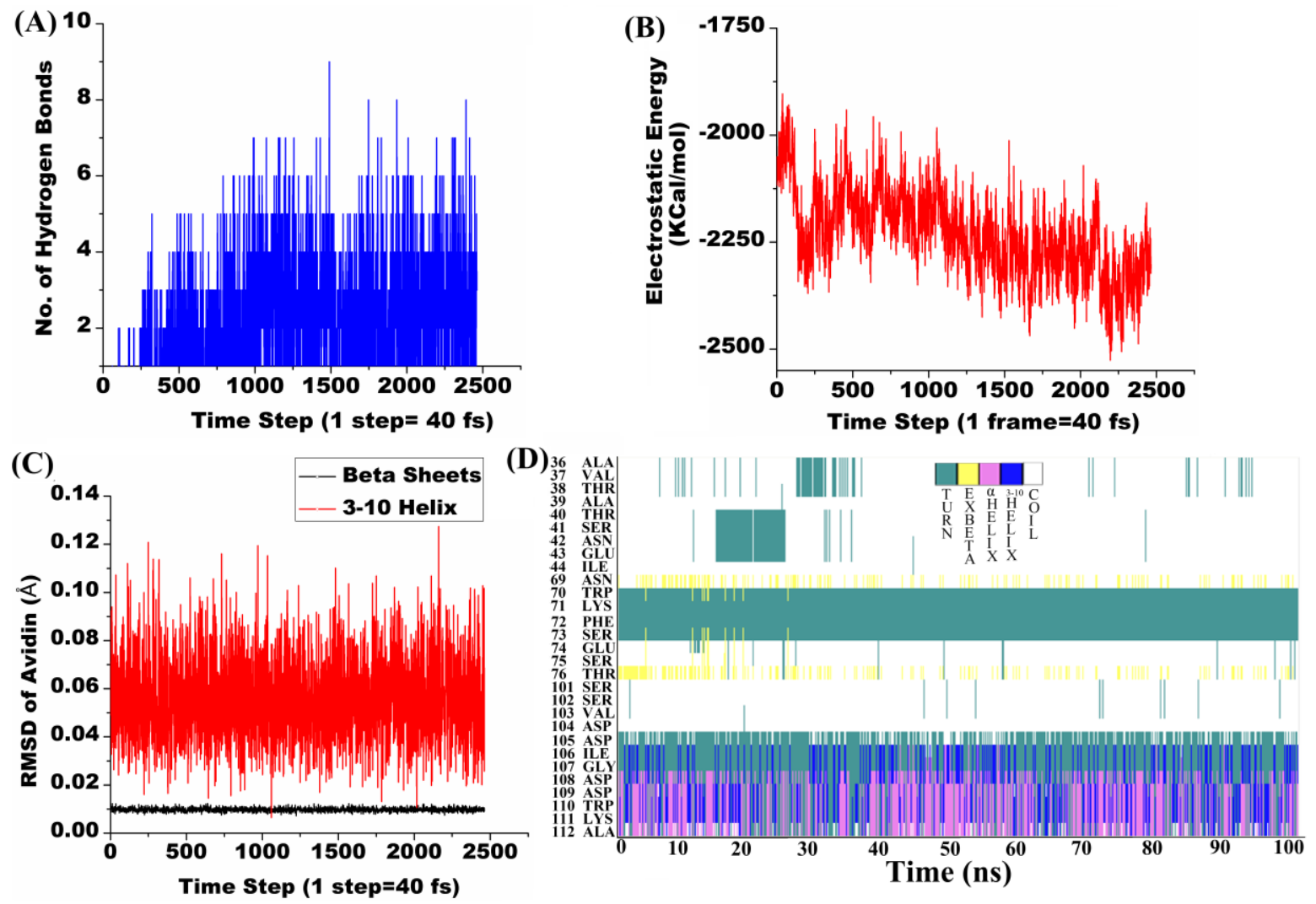

Fig. 4: (A) The number of hydrogen bonds between avidin and graphene sheet and their lifetimes as a function of time; (B) Electrostatic interaction between avidin and a single layer graphene sheet; (C) RMSD of the $\beta$ sheets and 3-10 helix of avidin over the entire time period of $100 \mathrm{~ns}$; (D) Secondary structure of avidin during the binding interactions with the graphene sheet showing no permanent changes to the avidin structure, which suggests that its function as a biotin binding protein is preserved.

\subsection{Fabrication of graphene polypyrrole nanocomposite}

Figures 5A and 5B illustrate the schematic for fabrication of the G-PPy nanocomposite substrate on a Cr-nickel surface and the final G-PPy nanocomposite film respectively. Due to the reduced content of pyrrole, the surface of the final nanocomposite film is more flake-like in appearance compared to the nanocomposite film synthesized by Aphale and colleagues (Aphale et al., 2015). This morphology is also evident upon surface examination of the new and conventional G-PPy substrates as seen from Figure 6. Figure 5C shows the EIS data for different stages of the experimental G-PPy-avidin substrate, which is coherent with the visual data. The initial bare and incomplete $\mathrm{Ni}$ - circuit yields a large impedance curve, which is significantly reduced upon addition of G-PPy, while addition of avidin to the surface slightly increases the total impedance of the system. 

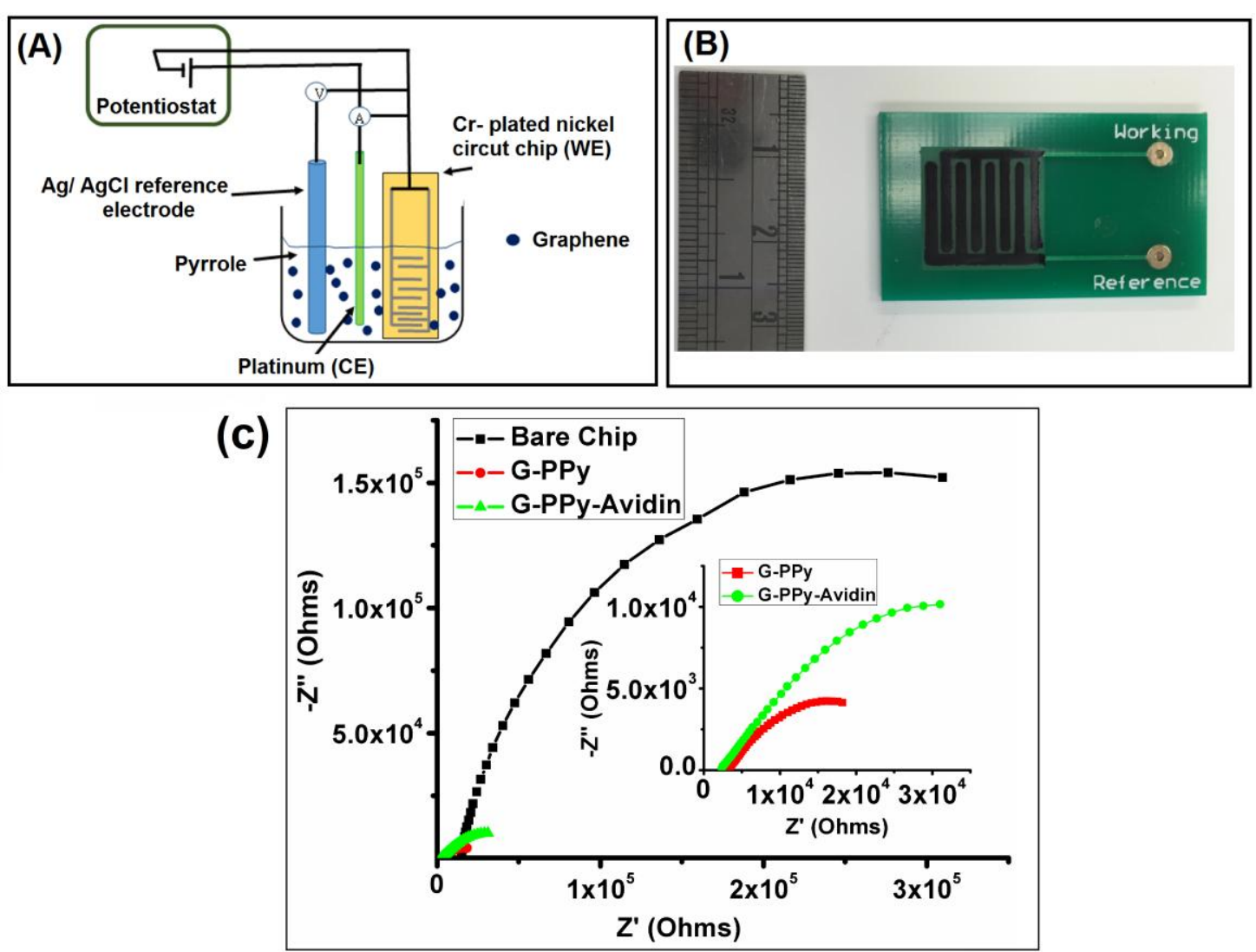

Fig. 5: (A) Schematic representation of the fabrication of graphene-polypyrrole nanocomposite surface; (B) Topview of experimental G-PPy nanocomposite film; (C) Average EIS analysis and comparison of bare surface, G-PPy and G-PPy-avidin surface 

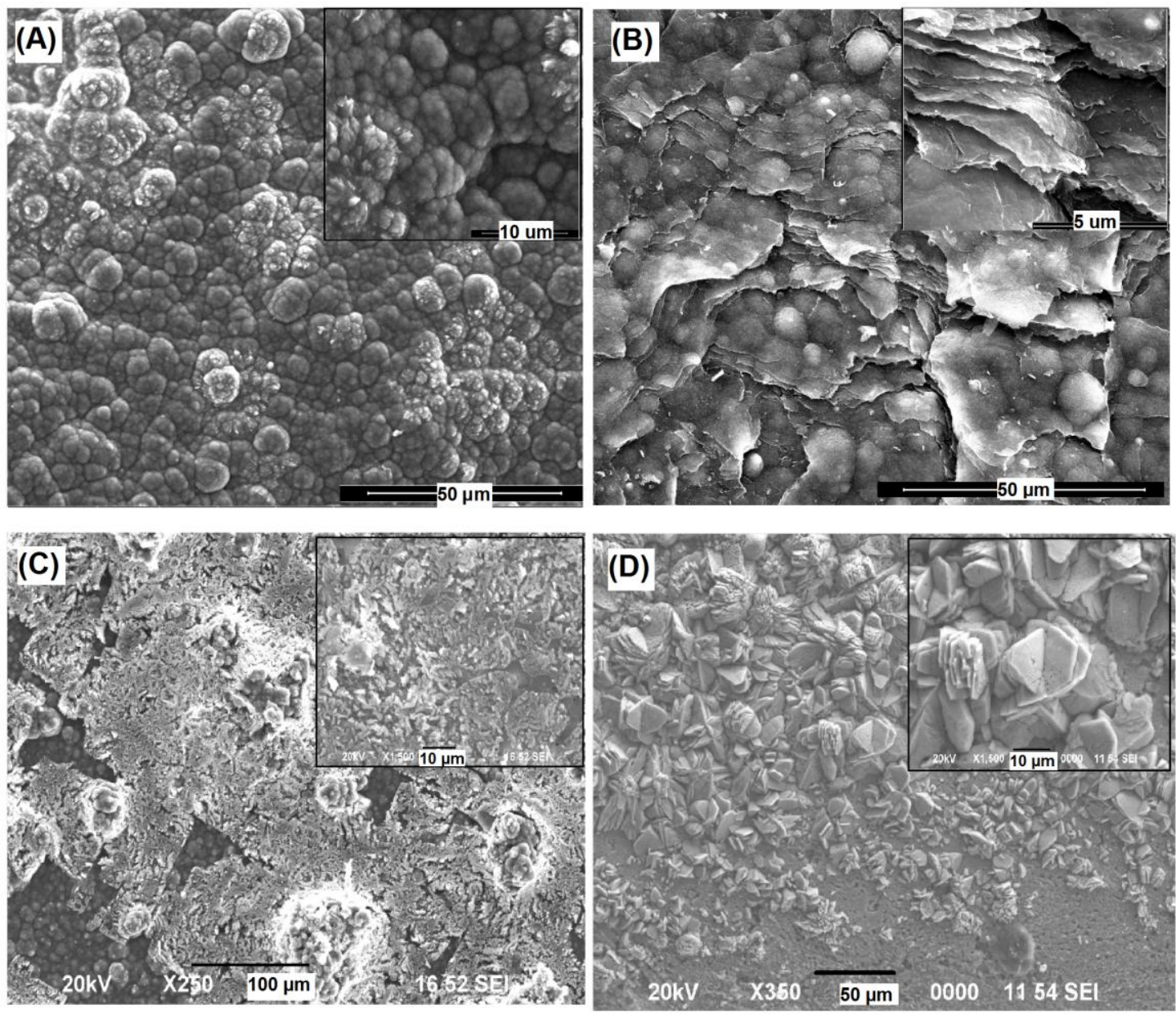

Fig. 6: SEM analysis of : (A) Control G-PPy surface; (B) High resolution image revealing only embedded graphene flakes; (C) Experimental G-PPy-avidin surface showing greater levels of exfoliated graphene; (D) High resolution image revealing graphene-flakes completely engulfed by avidin molecules.

SEM images of the surface of the control substrate, after soaking with avidin solution, reveal only predominant distribution of alveolar-like polypyrrole (Figure 6A). Upon further magnification, small graphene flakes can be seen embedded between the pyrrole molecules with no protein attachment (Figure 6B). This is in stark contrast to the experimental substrate where SEM images reveal a large quantity of exfoliated graphene flakes (Figure 6C). Further magnification reveals that the surface of these exposed graphene flakes has been completely engulfed with globular-like avidin molecules (Figure 6D), thus confirming our initial hypothesis that avidin in its native form can be deposited onto the graphene film without the need of any additional functionalization, thereby opening new directions for graphene based free standing biosensing films commonly used in avidin/biotin achoring mechanisms. 


\section{Discussion}

The primary objective of this study is fabrication and characterization of a free standing graphene film that serves as an interface between the molecular recognition element and the transducer. Based on molecular dynamics simulations and experimental analysis, we report an optimized graphenated polypyrrole film as a novel step towards formation of a substrate with anchored avidin for immobilization of biotinylated probes. Given previous research on the fabrication of G-PPy substrates and the possibility that pyrrole and avidin might not interact constructively to form a nanocomposite graphene film, we first investigated the interactions between a bare single layer graphene sheet and a single-barrel avidin monomer by MD to understand their association in the absence of pyrrole. The results revealed that avidin and graphene can form stable complexes primarily via hydrophobic forces with some contribution from van der Waals, electrostatic and hydrogen bonding interactions.

As seen in Figure 1, a perfectly barrel shaped avidin monomer goes through conformational rearrangement to spread near the surface of the graphene sheet, indicating adsorbtion on the surface to form a stable complex. In consecutive frames of the simulation, avidin moves closer to the surface of graphene present in a periodic water box indicating an attractive interaction. This avidin-mediated adsorption is further substantiated in Figure 2A, which shows that the distance between the center-of-mass of avidin and graphene decreases with time. A subsequent increase in the distance beyond the timeframe 1100 ( 1 step $=40 \mathrm{fs}$ ) indicates surface induced changes in avidin conformation by way of rearrangement of residues near the surface of graphene. For a quantitative assessment of the adsorption, the interaction energy (van der Waals+Electrostatic) between avidin and graphene was analyzed. Figure 2B indicates a decreasing trend in the interaction energy implying an increase in the effective interaction between avidin and graphene. The process of protein adsorption on a substrate is associated with slight conformational variations and residue rearrangement for more favorable interactions, hence the trend observed above is not linear. The residues of avidin may overcome an energy barrier before moving closer to the surface of graphene hovering at a distance closer than $5 \AA$ (typical adsorption distance) while adopting a favorable orientation for stronger interactions. If all of these changes are localized to the interface, they can contribute to maximum interaction and stability of avidin on the graphene surface, without a significant disruption in avidin conformation or loss of biotin binding function.

The RMSD data for avidin in the presence and absence of graphene, as shown in Figure $3 \mathrm{~A}$, reveals an important insight on the stability of the rapidly conforming residues. The deviation of the avidin monomer in the absence of graphene appears very large $(\sim 3 \AA)$ compared to only $0.5 \AA$ in the presence of graphene. This result indicates that graphene plays an important role in stabilizing the conformational changes of avidin residues by relaxing the oscillations resulting from the rearrangement. Comparison of Figures $3 \mathrm{~A}$ and $3 \mathrm{~B}$ reveals that the adsorption process starts at $\sim 50 \mathrm{~ns}$ and, as shown in Table 1 and Figure 3B, loops L3 ( $\beta 3-\beta 4)$ - residues 36 to 44 , L5 ( $\beta 5-\beta 6)$ - residues 69 to 76 and L7 ( $\beta 7-\beta 8)$ - residues 101 to 112 , participate in the binding process with the graphene sheet. Furthermore, the rapid conformational changes (larger RMSD) of loops L5 and L7 (Figure 3B) indicate these structural elements play a crucial role in the binding events. It is important to note that loop L3, which plays a vital role in binding biotin, is unaffected during and after the time of adsorption (Figure 3B shows that the L3 undergoes only minor fluctuations). The role of individual residues was further investigated by analyzing the interaction energy of the individual heavy atoms as well as hydrogen atoms of the adsorbed 
residues as a function of the cut-off distance from the surface of the graphene sheet, as shown in Figures 3C, 3D and 3E. As avidin moves closer to the surface of graphene, analysis of the number of atoms below $5 \AA$ distance from the graphene surface reveals that compared to distances between $3 \AA-5 \AA$, which are typical for strong VDW and moderate to weak hydrogen bonding interactions, distances between $2 \AA-3 \AA$ show only hydrogen atoms (Figure $3 \mathrm{C}$ inset). This finding indicates the dominance of hydrogen bonding interactions below $3 \AA$. Typically, the intermolecular forces between two binding molecules are dominant below the separation distance of $5 \AA$, hence this study underscores a cut-off distance below $5 \AA$ as the yardstick for the different binding events. According to the MD calculations there are close to 150 atoms from 19 residues below $5 \AA$ in the system, presenting a large enough surface area of adsorbtion on the graphene sheet and thereby substantiating the hypothesis that avidin and graphene can form a stable complex. As the interaction strength is determined by the attraction between the surface and the adjacent atoms, the interaction energy between avidin and graphene was divided by the number of adsorbed atoms as a function of cutoff distance (Figure 3E). The data show that the interaction energy per atom, both including and excluding hydrogen, has a sharp dip at a cut-off distance close to $4 \AA$, thereby indicating that the optimum adsorption distance for avidin on a single layer graphene sheet is $\sim 4 \AA$.

Hydrogen bonds below $3 \AA$ were analyzed further (Figure 4A), and the data show an increase in the number of hydrogen bonds with longer lifetimes, again highlighting the crucial role of this interaction during complex formation between between avidin and graphene. The interaction energy beyond a cut-off distance of $5 \AA$ (Figure 3D) also demonstrates the presence of long-range electrostatic attractive forces between avidin and graphene that increase by 375 $\mathrm{kCal} / \mathrm{mol}$ (Figure 4B) over a time span of $100 \mathrm{~ns}$. Indirectly, this can also be determined by comparing Figures $2 \mathrm{~A}, 3 \mathrm{~B}$ and 3D, where although the interaction energy stabilizes after $40 \mathrm{~ns}$ (1000 time steps), there is no adsorption of the residues until $60 \mathrm{~ns}$, again indicating the dominant role of electrostatics between $40 \mathrm{~ns}$ and $60 \mathrm{~ns}$. Our findings indicate that the adsorption of avidin on graphene can primarily be attributed to rearrangement of avidin residues and slight changes in conformation localized to the interface. It is important, however, to determine that the change in conformation does not lead to disruption in tertiary structure and loss of avidin function, which could render it incapable of binding biotin. One measure of adsorption and formation of stable complexes is the RMSD of the secondary structural elements ( $\beta$-sheets and helices) of a protein. Avidin is primarily composed of eight $\beta$ sheets whose RMSD demonstrate a very stable structure with deviations fluctuating around $0.01 \AA$ (Figure 4C). Apart from these $\beta$ sheets, a small section of the avidin molecule (residues 106-111) forms a 3-10 helix whose RMSD fluctuates around $0.06 \AA$ (Figure 4C); the slightly larger RMSD value suggests minor rearrangements in the 3-10 helix on interaction between graphene and avidin. Loops L3, L5 and L7 maintain their structure as shown in Figure 4D: residues 36 - 44 (loop L3) maintain their structure as a coil; residues 69 - 76 (loop L5) maintain their structure as a turn $(70-73)$ and a coil (74 - 75); residues 101 - 112 (loop L7) maintain their structure as a turn (101 - 104) and a 3-10 helix (106-111). The slight rearrangement of 3-10 helix residues appears to contribute to better adsorption of avidin monomer on the surface of graphene, as evident from a comparison of supplementary Figures S1A and S1B; in the absence of graphene (Figure S1A), the $\beta$ sheets $\beta 2$ and $\beta 6$ have $\sim 50 \%$ deterioration, the biotin binding loop L3 has a conformational change from a coil to a turn, and the 3-10 helix reconforms to an $\alpha$-helix. This rupture of $\beta$-sheets and large conformational changes especially of the 3-10 helix, would compromise the stability and more importantly the function of avidin. Thus, the formation of avidin/graphene nanocomposite does appear to induce conformational changes that can stabilize avidin on the surface of graphene and 
at the same time retain its function in binding biotin, which is crucial for the performance of the biosensor device. Finally, these MD analyses informed the synthesis of a free standing graphene film with a minimum concentration of pyrrole (compared to Aphale and colleagues, (Aphale et al., 2015)) as avidin is found to interact with graphene in a both stable and energetically favorable way.

The computational results are further verified through both electrochemical and visual analysis. Morphological examination of the experimental G-PPy-avidin complex surface via SEM shows that the avidin molecules cluster and completely cloak the surface of the graphene flakes, thus supporting our simulation data. EIS data of individual stages during development of the G-PPy-avidin substrate yielded characteristic impedance curves, with the bare chip having the highest impedance and the G-PPy nanocomposite surface having the least impedance under the same conditions. The reduction in impedance is due to the combined conductive properties of graphene and polypyrrole. Protein attachment leads to a significant increase in impedance due to increase in mass, confirming the attachment of the avidin molecules to the exfoliated graphene flakes on the G-PPy nanocomposite surface. However, the total impedance remains significantly less compared to that of the bare chip. All of the electrochemical data confirm that total impedance of a system is characteristic to the components of the particular system, thereby validating the presence of each component. Addition of further components, such as oligonucleotides or biotinylated probes is therefore expected to yield different data, allowing label-free detection of biomolecules. Thus, our data support the conclusion that the fabricated GPPy-avidin complex could serve as an efficient substrate for both label-free detection and point quantification of biomolecules present at low concentrations in solution. Following the success of the characterization of the G-PPy-avidin complex, our experimental substrate is currently being analyzed for DNA deposition and possible detection of DNA mismatch repair proteins. It is thus expected that the presence of graphene on any substrate acting as a biological recognition element can extensively exploit the high affinity between avidin and biotin as a powerful and indispensable tool in biosensor applications. 


\section{Conclusion}

This is the first reported study on direct interaction between graphene and avidin. We have examined the interactions and energy changes involved in the adsorption of avidin on a 2dimensional graphene surface by both computational and experimental analysis. The experimental data demonstrate selective adsorption of avidin molecules on the surface of graphene flakes (SEM) and a difference in electrical impedance between a 'G-PPy microelectrode chip' and 'avidin-G-PPy microelectrode chip' (EIS), indicating successful deposition of avidin on the graphene surface as predicted by the MD data.

\section{Acknowledgements}

This research was supported by a grant from the Connecticut Department of Public Health to M.M.H. and P.P. (DPH Log \#2014-0136). We would like to thank Jeff Gilarde, Director of Confocal Imaging at Wesleyan University, for conducting the SEM analysis.

\section{References}

Alava, T., Mann, J.A., Théodore, C., Benitez, J.J., Dichtel, W.R., Parpia, J.M., Craighead, H.G., 2013. Control of the Graphene-Protein Interface Is Required To Preserve Adsorbed Protein Function. Anal. Chem. 85, 2754-2759. doi:10.1021/ac303268z

Aphale, A., Maisuria, K., Mahapatra, M.K., Santiago, A., Singh, P., Patra, P., 2015. Hybrid Electrodes by In-Situ Integration of Graphene and Carbon-Nanotubes in Polypyrrole for Supercapacitors. Sci. Rep. 5, 14445. doi:10.1038/srep14445

Daniels, J.S., Pourmand, N., 2007. Label-Free Impedance Biosensors: Opportunities and Challenges. Electroanalysis 19, 1239-1257. doi:10.1002/elan.200603855

Drummond, T.G., Hill, M.G., Barton, J.K., 2003. Electrochemical DNA sensors. Nat. Biotechnol. 21, 1192-9. doi:10.1038/nbt873

Dupont-Filliard, A., Billon, M., Livache, T., Guillerez, S., 2004. Biotin/avidin system for the generation of fully renewable DNA sensor based on biotinylated polypyrrole film. Anal. Chim. Acta 515, 271-277. doi:10.1016/j.aca.2004.03.072

Dupont-Filliard, A., Roget, A., Livache, T., Billon, M., 2001. Reversible oligonucleotide immobilisation based on biotinylated polypyrrole film. Anal. Chim. Acta 449, 45-50. doi:10.1016/S0003-2670(01)01339-3

Evans, E., Ritchie, K., 1997. Dynamic strength of molecular adhesion bonds. Biophys. J. 72, 1541-1555. doi:10.1016/S0006-3495(97)78802-7

Georgakilas, V., Otyepka, M., Bourlinos, A.B., Chandra, V., Kim, N., Kemp, K.C., Hobza, P., Zboril, R., Kim, K.S., 2012. Functionalization of graphene: covalent and non-covalent approaches, derivatives and applications. Chem. Rev. 112, 6156-214.

doi:10.1021/cr3000412 
Gräslund, S., Nordlund, P., Weigelt, J., Hallberg, B.M., Bray, J., Gileadi, O., Knapp, S., Oppermann, U., Arrowsmith, C., Hui, R., Ming, J., dhe-Paganon, S., Park, H., Savchenko, A., Yee, A., Edwards, A., Vincentelli, R., Cambillau, C., Kim, R., Kim, S.-H., Rao, Z., Shi, Y., Terwilliger, T.C., Kim, C.-Y., Hung, L.-W., Waldo, G.S., Peleg, Y., Albeck, S., Unger, T., Dym, O., Prilusky, J., Sussman, J.L., Stevens, R.C., Lesley, S.A., Wilson, I.A., Joachimiak, A., Collart, F., Dementieva, I., Donnelly, M.I., Eschenfeldt, W.H., Kim, Y., Stols, L., Wu, R., Zhou, M., Burley, S.K., Emtage, J.S., Sauder, J.M., Thompson, D., Bain, K., Luz, J., Gheyi, T., Zhang, F., Atwell, S., Almo, S.C., Bonanno, J.B., Fiser, A., Swaminathan, S., Studier, F.W., Chance, M.R., Sali, A., Acton, T.B., Xiao, R., Zhao, L., Ma, L.C., Hunt, J.F., Tong, L., Cunningham, K., Inouye, M., Anderson, S., Janjua, H., Shastry, R., Ho, C.K., Wang, D., Wang, H., Jiang, M., Montelione, G.T., Stuart, D.I., Owens, R.J., Daenke, S., Schütz, A., Heinemann, U., Yokoyama, S., Büssow, K., Gunsalus, K.C., 2008. Protein production and purification. Nat. Methods 5, 135-46. doi:10.1038/nmeth.f.202

Gu, Z., Yang, Z., Wang, L., Zhou, H., Jimenez-Cruz, C.A., Zhou, R., 2015. The role of basic residues in the adsorption of blood proteins onto the graphene surface. Sci. Rep. 5, 10873. doi:10.1038/srep10873

Haddad, R., Cosnier, S., Maaref, A., Holzinger, M., 2009. Non-covalent biofunctionalization of single-walled carbon nanotubes via biotin attachment by $\pi$-stacking interactions and pyrrole polymerization. Analyst 134, 2412-8. doi:10.1039/b916774a

Hu, J., Ruan, X., Chen, Y.P., 2009. Thermal conductivity and thermal rectification in graphene nanoribbons: a molecular dynamics study. Nano Lett. 9, 2730-5. doi:10.1021/n1901231s

Hu, W., Peng, C., Lv, M., Li, X., Zhang, Y., Chen, N., Fan, C., Huang, Q., 2011. Protein coronamediated mitigation of cytotoxicity of graphene oxide. ACS Nano 5, 3693-700. doi:10.1021/nn200021j

Humphrey, W., Dalke, A., Schulten, K., 1996. VMD: Visual molecular dynamics. J. Mol. Graph. 14, 33-38. doi:10.1016/0263-7855(96)00018-5

Isralewitz, B., Gao, M., Schulten, K., 2001. Steered molecular dynamics and mechanical functions of proteins. Curr. Opin. Struct. Biol. 11, 224-230. doi:10.1016/S0959440X(00)00194-9

Izrailev, S., Stepaniants, S., Balsera, M., Oono, Y., Schulten, K., 1997. Molecular dynamics study of unbinding of the avidin-biotin complex. Biophys. J. 72, 1568-81. doi:10.1016/S0006-3495(97)78804-0

Jeffrey, G.A., 1997. An Introduction to Hydrogen Bonding. Oxford University Press. Jiang, J.-W., Wang, J.-S., Li, B., 2009. Young's modulus of graphene: A molecular dynamics 
study. Phys. Rev. B 80, 113405. doi:10.1103/PhysRevB.80.113405

Jorgensen, W.L., Chandrasekhar, J., Madura, J.D., Impey, R.W., Klein, M.L., 1983. Comparison of simple potential functions for simulating liquid water. J. Chem. Phys. 79, 926. doi: $10.1063 / 1.445869$

Kamiya, Y., Yamazaki, K., Ogino, T., 2014. Protein adsorption to graphene surfaces controlled by chemical modification of the substrate surfaces. J. Colloid Interface Sci. 431, 77-81. doi:10.1016/j.jcis.2014.06.023

Kostarelos, K., Novoselov, K.S., 2014. Exploring the Interface of Graphene and Biology. Sci. 344, 261-263. doi:10.1126/science. 1246736

Kuila, T., Bose, S., Khanra, P., Mishra, A.K., Kim, N.H., Lee, J.H., 2011. Recent advances in graphene-based biosensors. Biosens. Bioelectron. 26, 4637-48.

doi:10.1016/j.bios.2011.05.039

Lee, W.H., Park, J., Kim, Y., Kim, K.S., Hong, B.H., Cho, K., 2011. Control of graphene fieldeffect transistors by interfacial hydrophobic self-assembled monolayers. Adv. Mater. 23, 3460-4. doi:10.1002/adma.201101340

Lu, C.-H., Yang, H.-H., Zhu, C.-L., Chen, X., Chen, G.-N., 2009. A Graphene Platform for Sensing Biomolecules. Angew. Chemie Int. Ed. 48, 4785-4787. doi:10.1002/anie.200901479

MacKerell, A.D., Bashford, D., Dunbrack, R.L., Evanseck, J.D., Field, M.J., Fischer, S., Gao, J., Guo, H., Ha, S., Joseph-McCarthy, D., Kuchnir, L., Kuczera, K., Lau, F.T.K., Mattos, C., Michnick, S., Ngo, T., Nguyen, D.T., Prodhom, B., Reiher, W.E., Roux, B., Schlenkrich, M., Smith, J.C., Stote, R., Straub, J., Watanabe, M., Wiórkiewicz-Kuczera, J., Yin, D., Karplus, M., 1998. All-Atom Empirical Potential for Molecular Modeling and Dynamics Studies of Proteins $\uparrow$. J. Phys. Chem. B 102, 3586-3616. doi:10.1021/jp973084f

Nel, A.E., Mädler, L., Velegol, D., Xia, T., Hoek, E.M. V, Somasundaran, P., Klaessig, F., Castranova, V., Thompson, M., 2009. Understanding biophysicochemical interactions at the nano-bio interface. Nat. Mater. 8, 543-57. doi:10.1038/nmat2442

Ouerghi, O., Touhami, A., Jaffrezic-Renault, N., Martelet, C., Ouada, H.B., Cosnier, S., 2002. Impedimetric immunosensor using avidin-biotin for antibody immobilization. Bioelectrochemistry 56, 131-133. doi:10.1016/S1567-5394(02)00029-4

Pei, Q.X., Zhang, Y.W., Shenoy, V.B., 2010. A molecular dynamics study of the mechanical properties of hydrogen functionalized graphene. Carbon N. Y. 48, 898-904. doi:10.1016/j.carbon.2009.11.014

Phillips, J.C., Braun, R., Wang, W., Gumbart, J., Tajkhorshid, E., Villa, E., Chipot, C., Skeel, 
R.D., Kalé, L., Schulten, K., 2005. Scalable molecular dynamics with NAMD. J. Comput. Chem. 26, 1781-802. doi:10.1002/jcc.20289

Rodrigo, D., Limaj, O., Janner, D., Etezadi, D., Garcia de Abajo, F.J., Pruneri, V., Altug, H., 2015. Mid-infrared plasmonic biosensing with graphene. Science (80-. ). 349, 165-168. doi:10.1126/science.aab2051

Russo, C.J., Passmore, L.A., 2014. Controlling protein adsorption on graphene for cryo-EM using low-energy hydrogen plasmas. Nat. Methods 11, 649-652. doi:10.1038/nmeth.2931

Sadik, O.A., Aluoch, A.O., Zhou, A., 2009. Status of biomolecular recognition using electrochemical techniques. Biosens. Bioelectron. 24, 2749-65. doi:10.1016/j.bios.2008.10.003

Sarikaya, M., Tamerler, C., Jen, A.K.-Y., Schulten, K., Baneyx, F., 2003. Molecular biomimetics: nanotechnology through biology. Nat. Mater. 2, 577-85. doi:10.1038/nmat964

Shao, Y., Wang, J., Wu, H., Liu, J., Aksay, I.A., Lin, Y., 2010. Graphene Based Electrochemical Sensors and Biosensors: A Review. Electroanalysis 22, 1027-1036. doi:10.1002/elan.200900571

Tiwari, A.K., Roy, H.K., Lynch, H.T., 2015. Lynch syndrome in the 21st century: clinical perspectives. QJM. doi:10.1093/qjmed/hcv137

Wilchek, M., Bayer, E.A., 1988. The avidin-biotin complex in bioanalytical applications. Anal. Biochem. 171, 1-32.

Xue, T., Peng, B., Xue, M., Zhong, X., Chiu, C.-Y., Yang, S., Qu, Y., Ruan, L., Jiang, S., Dubin, S., Kaner, R.B., Zink, J.I., Meyerhoff, M.E., Duan, X., Huang, Y., 2014. Integration of molecular and enzymatic catalysts on graphene for biomimetic generation of antithrombotic species. Nat. Commun. 5, 3200. doi:10.1038/ncomms4200

Yokota, K., Takai, K., Enoki, T., 2011. Carrier Control of Graphene Driven by the Proximity Effect of Functionalized Self-assembled Monolayers. Nano Lett. 11, 3669-3675. doi: $10.1021 / \mathrm{nl} 201607 \mathrm{t}$

Zhang, J., Landry, M.P., Barone, P.W., Kim, J.-H., Lin, S., Ulissi, Z.W., Lin, D., Mu, B., Boghossian, A.A., Hilmer, A.J., Rwei, A., Hinckley, A.C., Kruss, S., Shandell, M.A., Nair, N., Blake, S., Şen, F., Şen, S., Croy, R.G., Li, D., Yum, K., Ahn, J.-H., Jin, H., Heller, D.A., Essigmann, J.M., Blankschtein, D., Strano, M.S., 2013. Molecular recognition using corona phase complexes made of synthetic polymers adsorbed on carbon nanotubes. Nat. Nanotechnol. 8, 959-68. doi:10.1038/nnano.2013.236

Zhang, M., Liao, C., Mak, C.H., You, P., Mak, C.L., Yan, F., 2015. Highly sensitive glucose sensors based on enzyme-modified whole-graphene solution-gated transistors. Sci. Rep. 5, 
8311. doi:10.1038/srep08311

Zhao, Y., Xing, G., Chai, Z., 2008. Nanotoxicology: Are carbon nanotubes safe? Nat. Nanotechnol. 3, 191-2. doi:10.1038/nnano.2008.77

Zhong, W.-R., Zhang, M.-P., Ai, B.-Q., Zheng, D.-Q., 2011. Chirality and thickness-dependent thermal conductivity of few-layer graphene: A molecular dynamics study. Appl. Phys. Lett. 98, 113107. doi:10.1063/1.3567415 
Dear Reviewer,

We would like to present to you our findings on the interaction of avidin protein with a pristine single layer of atomically thin graphene. This article titled, "INTERACTIONS BETWEEN AVIDIN AND GRAPHENE FOR DEVELOPMENT OF A BIOSENSING PLATFORM", describes a unique way that avidin interacts with a $88 \mathrm{X} 122$ angstrom single layer graphene sheet. It is further realized that the binding events are dominated by van der Waals and electrostatic interactions including hydrophobic and hydrogen bonding interactions below 4 angstrom. The binding events are simulated using Visual Molecular Dynamics (VMD) and the associated tool NAMD. It is also found that the secondary structure of avidin is preserved through the formation of the avidin/graphene complex and the EIS and SEM analysis of avidin coated free standing graphene film agrees with the simulated data. Therefore, a unique avidin-graphene based platform without additional functionalization opens up possibilities to design biosensors capable of detecting biotinylated macromolecules.

This is the first reported study on direct interaction between graphene and avidin. We have examined the interactions and energy changes involved in the adsorption of avidin on a 2dimensional graphene surface by both computational and experimental analysis. The experimental data demonstrate selective adsorption of avidin molecules on the surface of graphene flakes (SEM) and a difference in electrical impedance between a 'G-PPy microelectrode chip' and 'avidin-G-PPy microelectrode chip' (EIS), indicating successful deposition of avidin on the graphene surface as predicted by the MD data.

It is also found that individual avidin monomer can be stabilized using graphene as a support substrate. Based on the MD data, it is confirmed that a single avidin monomer would not loose its primary function as a biotin binding protein on adsorption of a graphene substrate.

Hope you find our results interesting and the manuscript in accordance with the scientific principles to expand our understanding of the avidin/graphene based biosensors.

Regards,

Prabir Patra

Isaac Macwan 
Dear Editor, Biosensors and Bioelectronics,

We would like to present to you our findings on the interaction of avidin protein with a pristine single layer of atomically thin graphene. This article titled, "INTERACTIONS BETWEEN AVIDIN AND GRAPHENE FOR DEVELOPMENT OF A BIOSENSING PLATFORM", describes a unique way that avidin interacts with a $88 \times 122$ angstrom single layer graphene sheet. It is further realized that the binding events are dominated by van der Waals and electrostatic interactions including hydrophobic and hydrogen bonding interactions below 4 angstrom. The binding events are simulated using Visual Molecular Dynamics (VMD) and the associated tool NAMD. It is also found that the secondary structure of avidin is preserved through the formation of the avidin/graphene complex and the EIS and SEM analysis of avidin coated free standing graphene film agrees with the simulated data. Therefore, a unique avidin-graphene based platform without additional functionalization opens up possibilities to design biosensors capable of detecting biotinylated macromolecules.

Our manuscript contains five colored figures and an SEM figure. In each case color is imperative to understand the sections pertaining to them. For example in figure 1 we are denoting each domain and respective protein residues with colors, in figure $3 a$, RMSD of avidin monomer in the presence and absence of graphene is shown in black and red colors, in figures $3 c, 3 d$ and $3 e$, the inclusion and exclusion of hydrogen atoms is shown using two different colors, figure $4 b$ shows the RMSD of beta sheets and 3-10 helix of the avidin monomer in red and black colors and figures $4 \mathrm{c}$ supplementary figure S1 show a colored distinction between the different secondary structures of the avidin monomer in the presence and absence of graphene.

Hope you find our results interesting and the manuscript in appropriate shape and format to be considered for your journal.

Regards,

Prabir Patra

Isaac Macwan 
Flick here to download high resolution image
Cls

(A) Ons

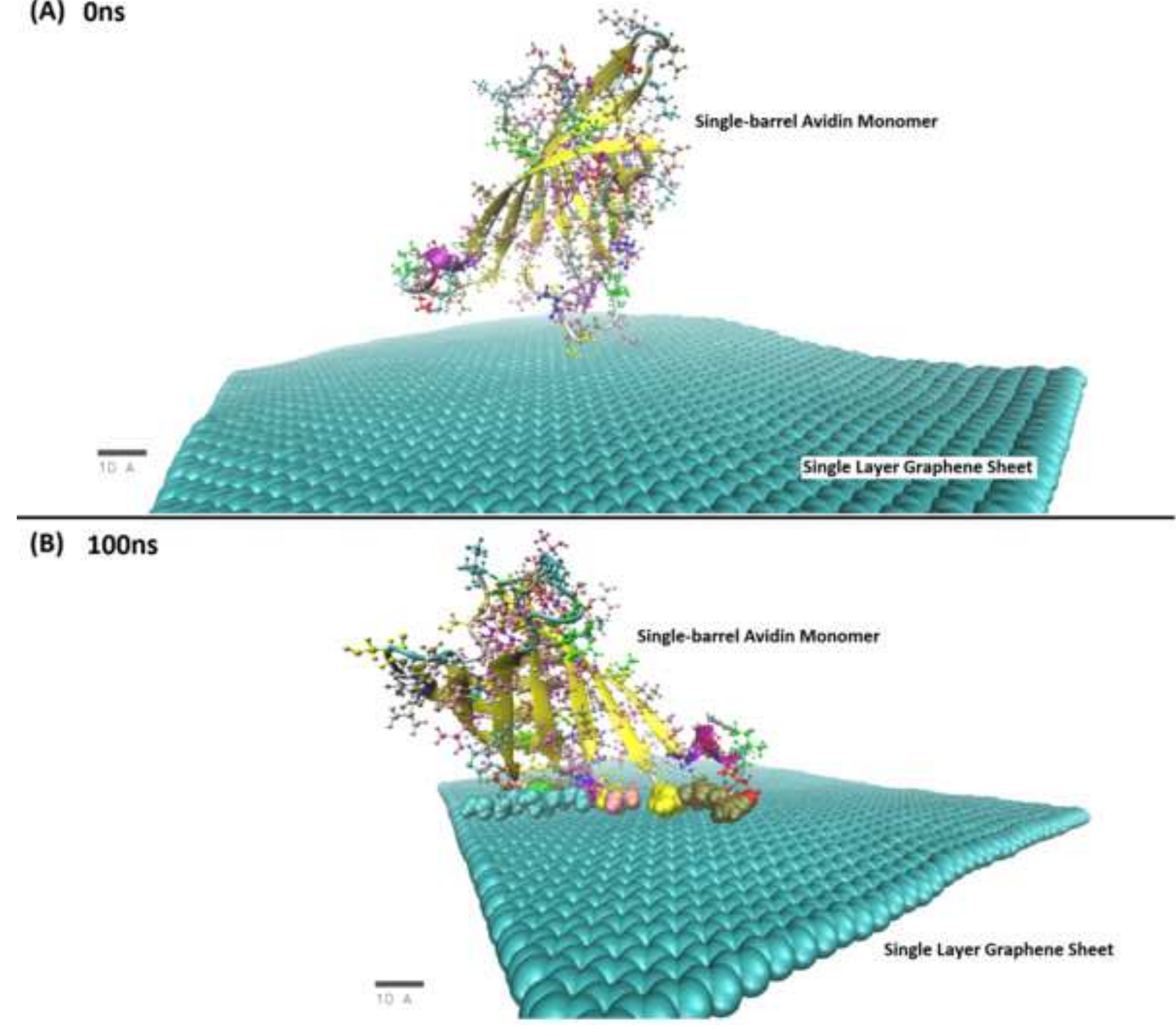

(B) $100 \mathrm{~ns}$

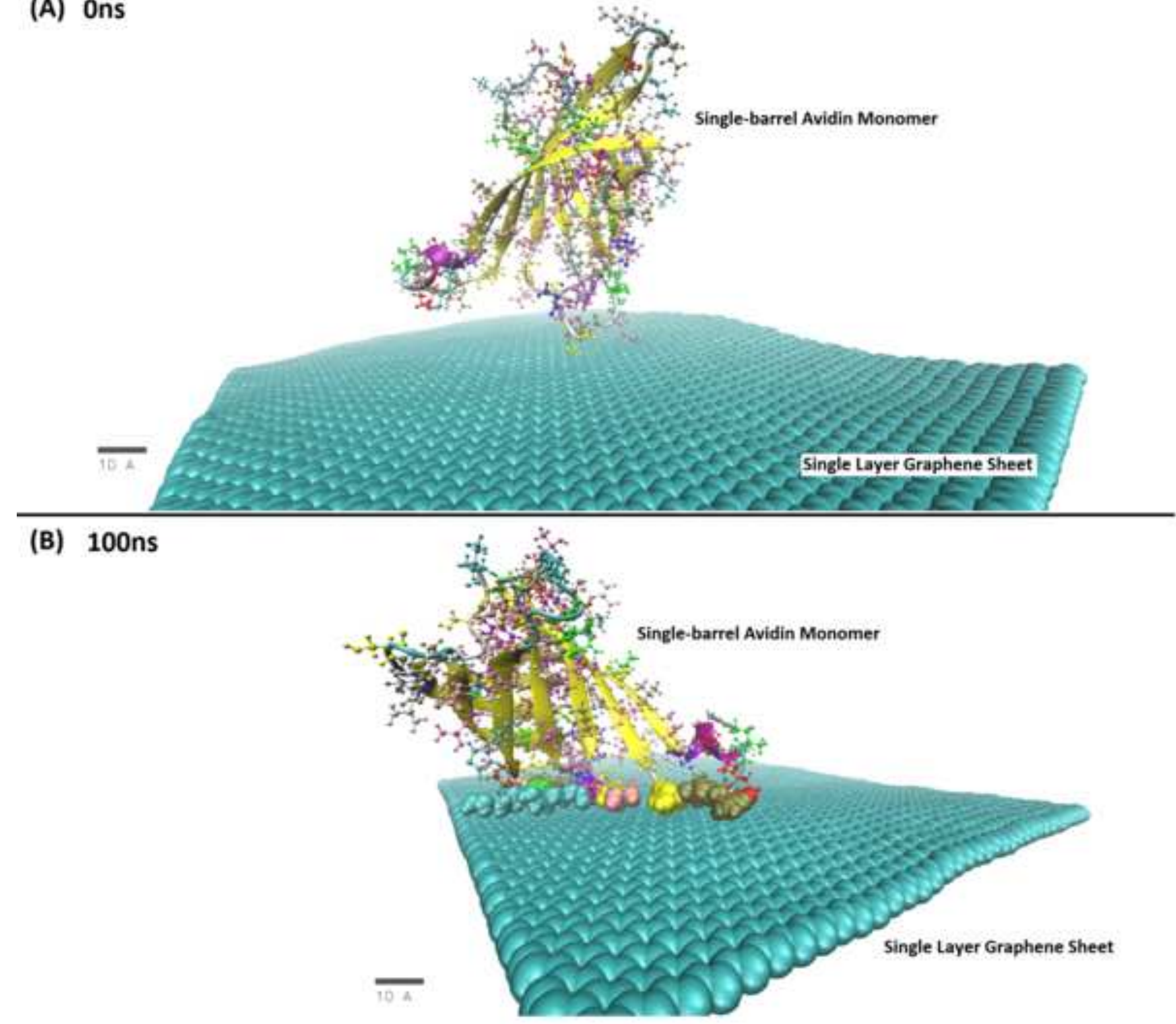



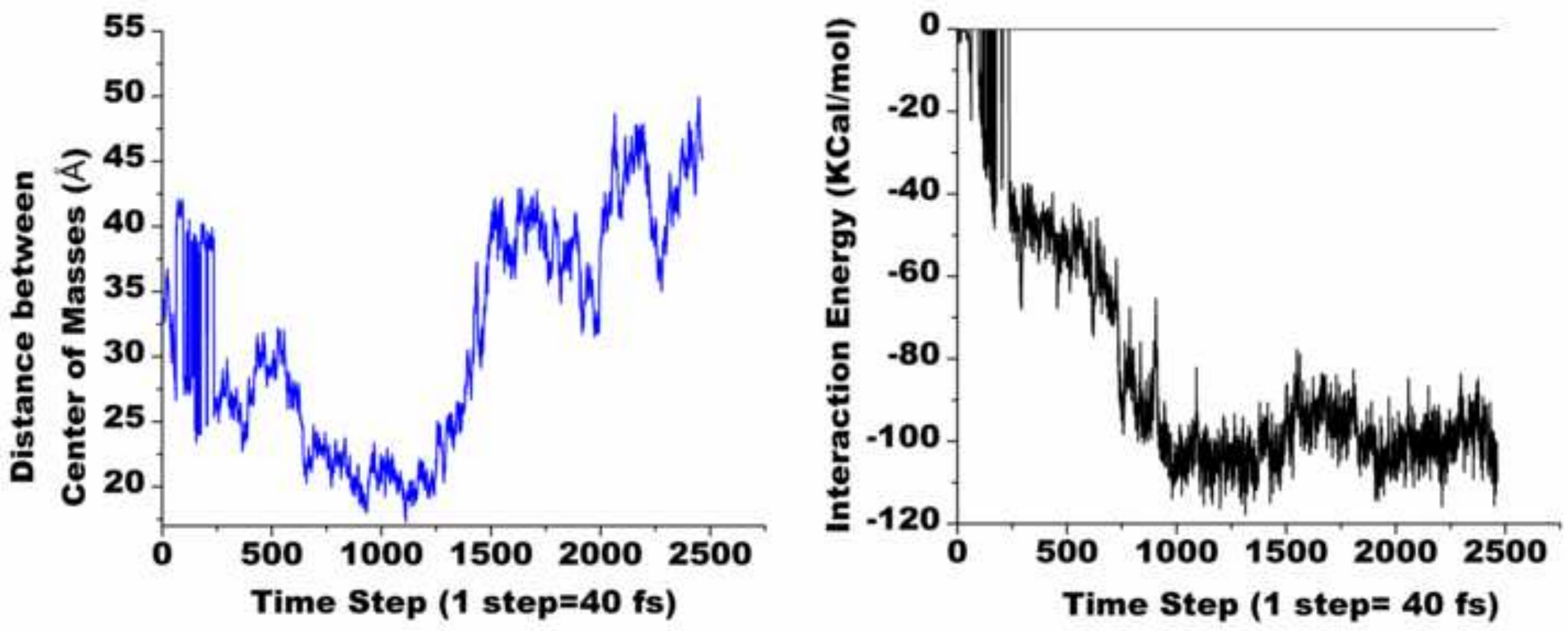
(A)
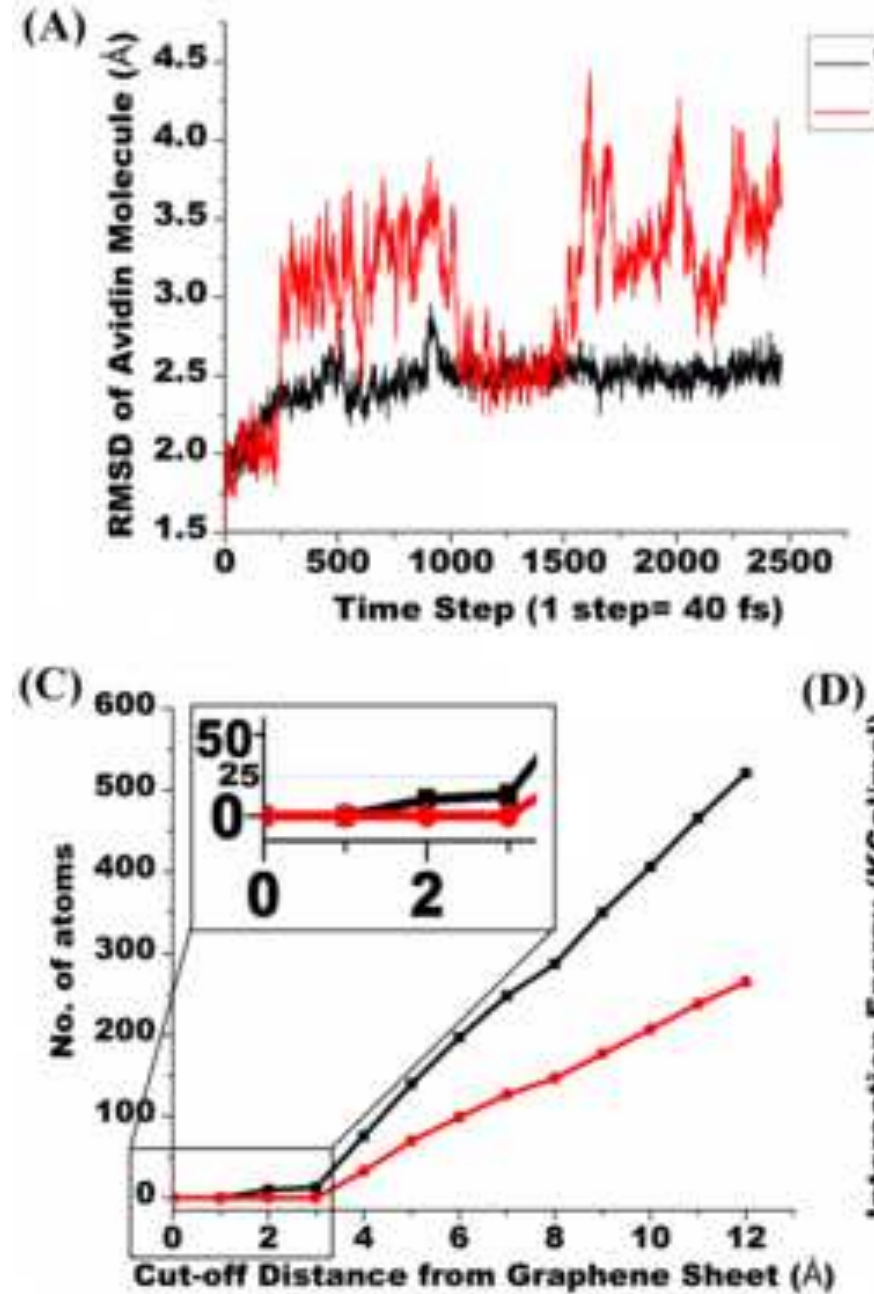

(D)

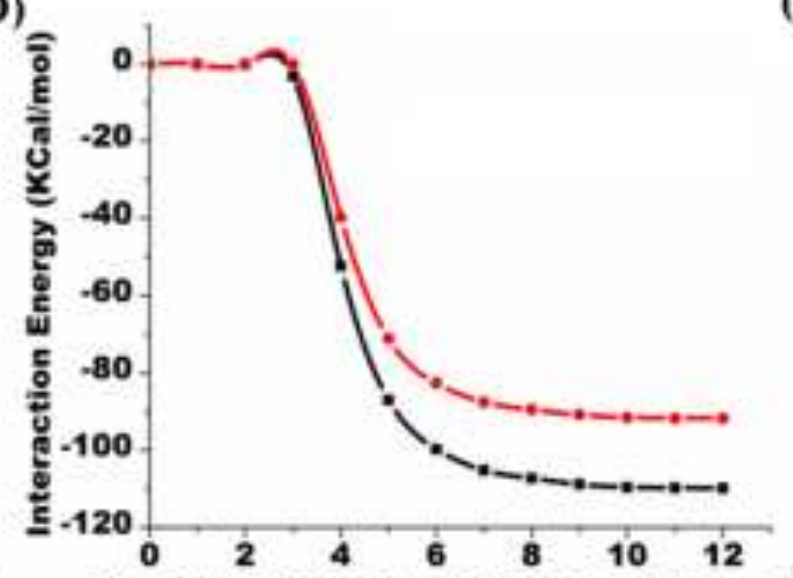

Cut off Distance from Graphene Surface (A)

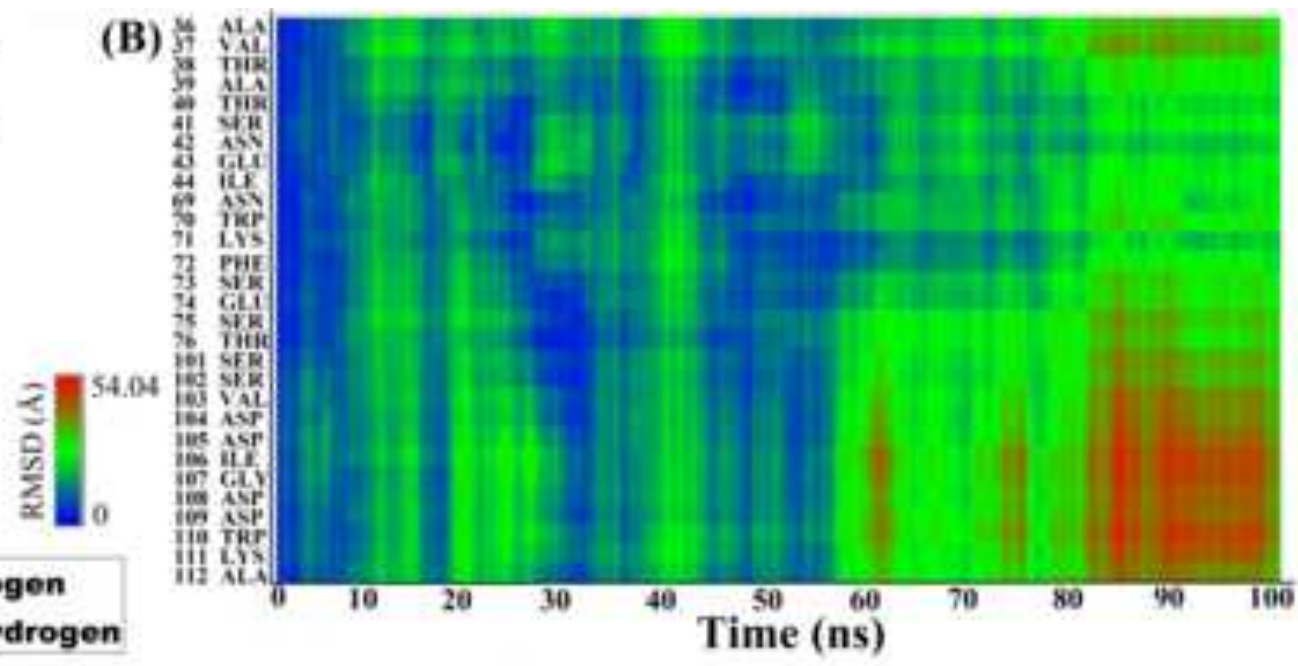

(E)

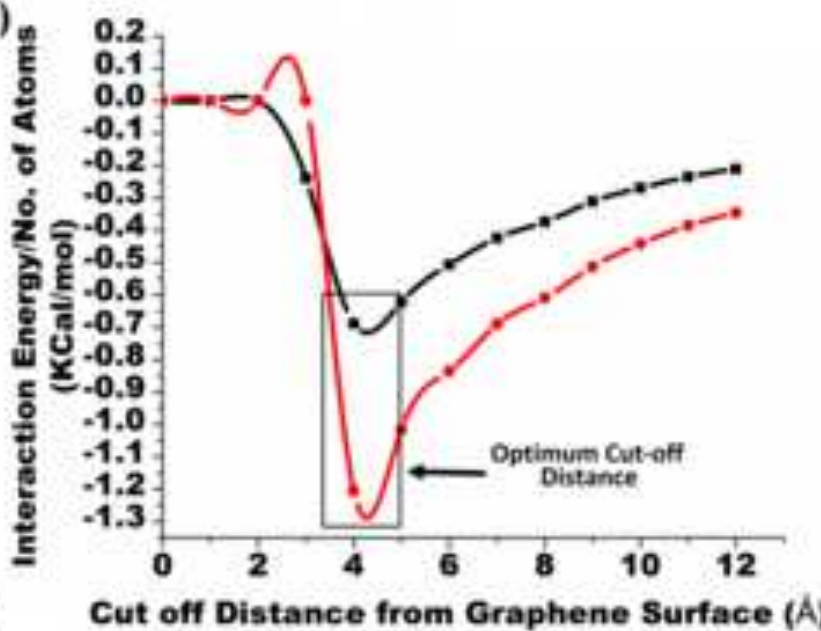



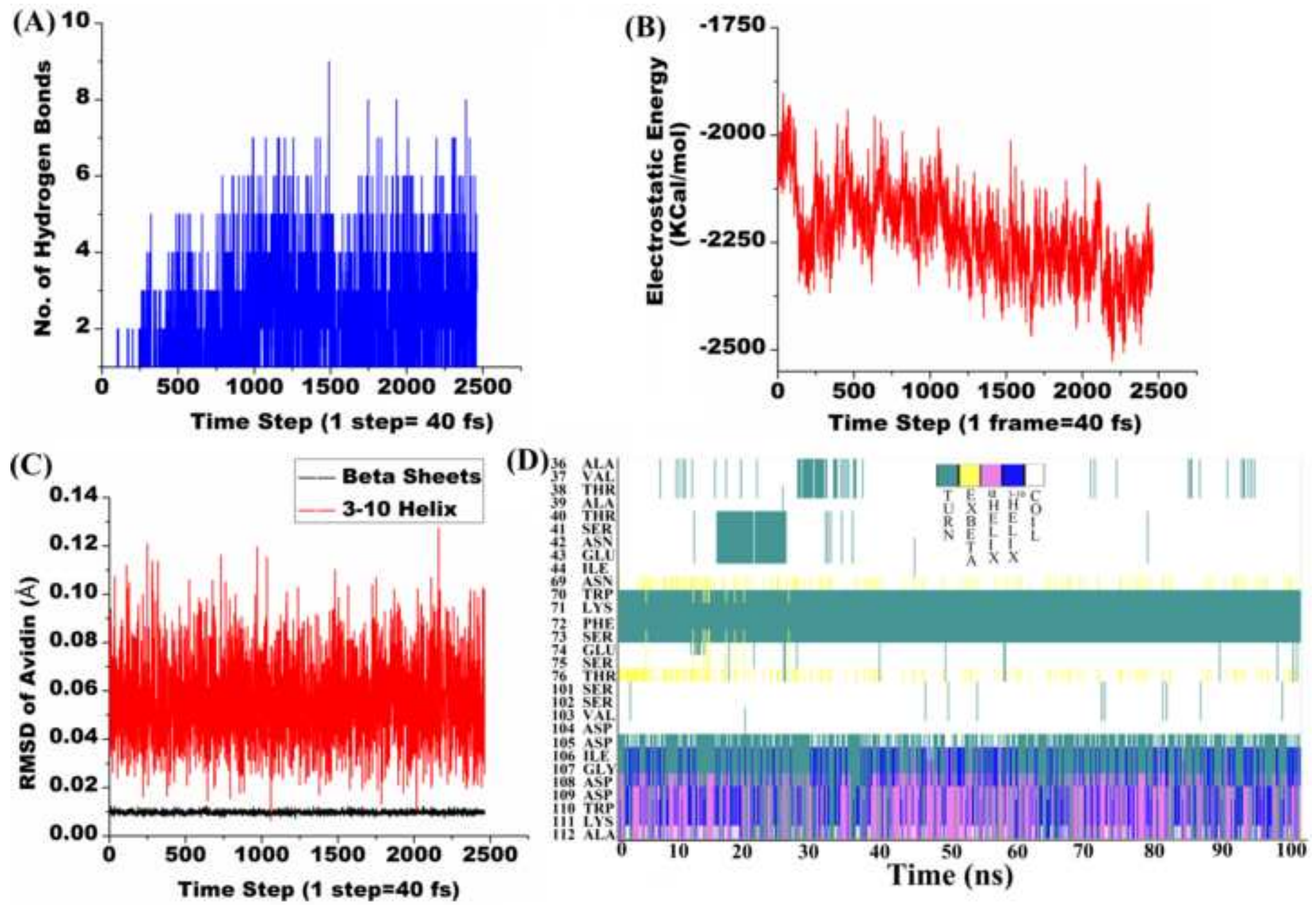
Click here to download high resolution image

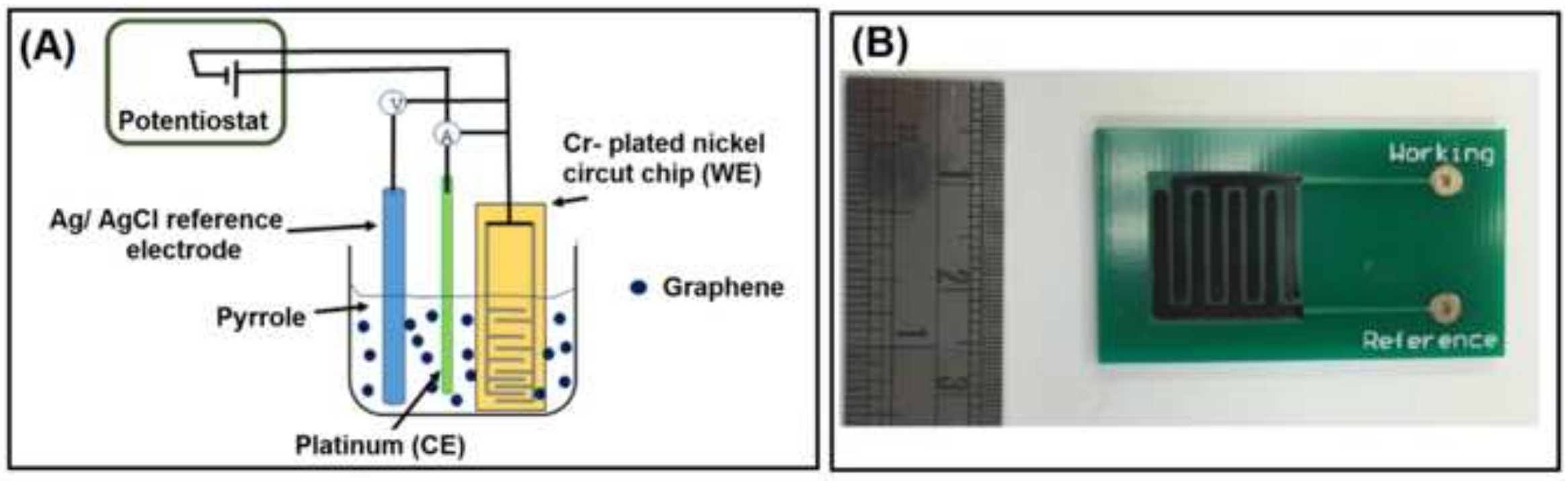

(c)

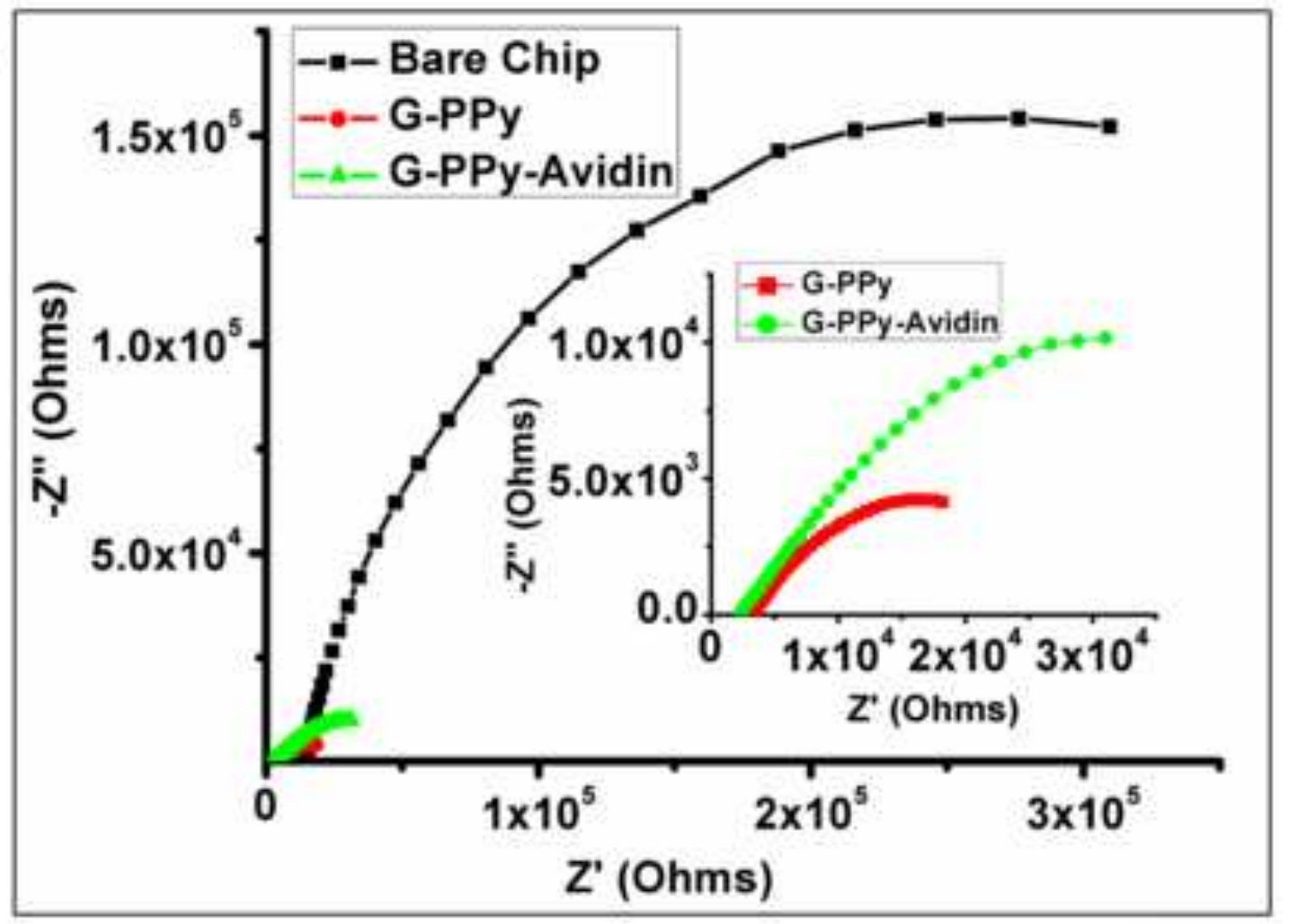



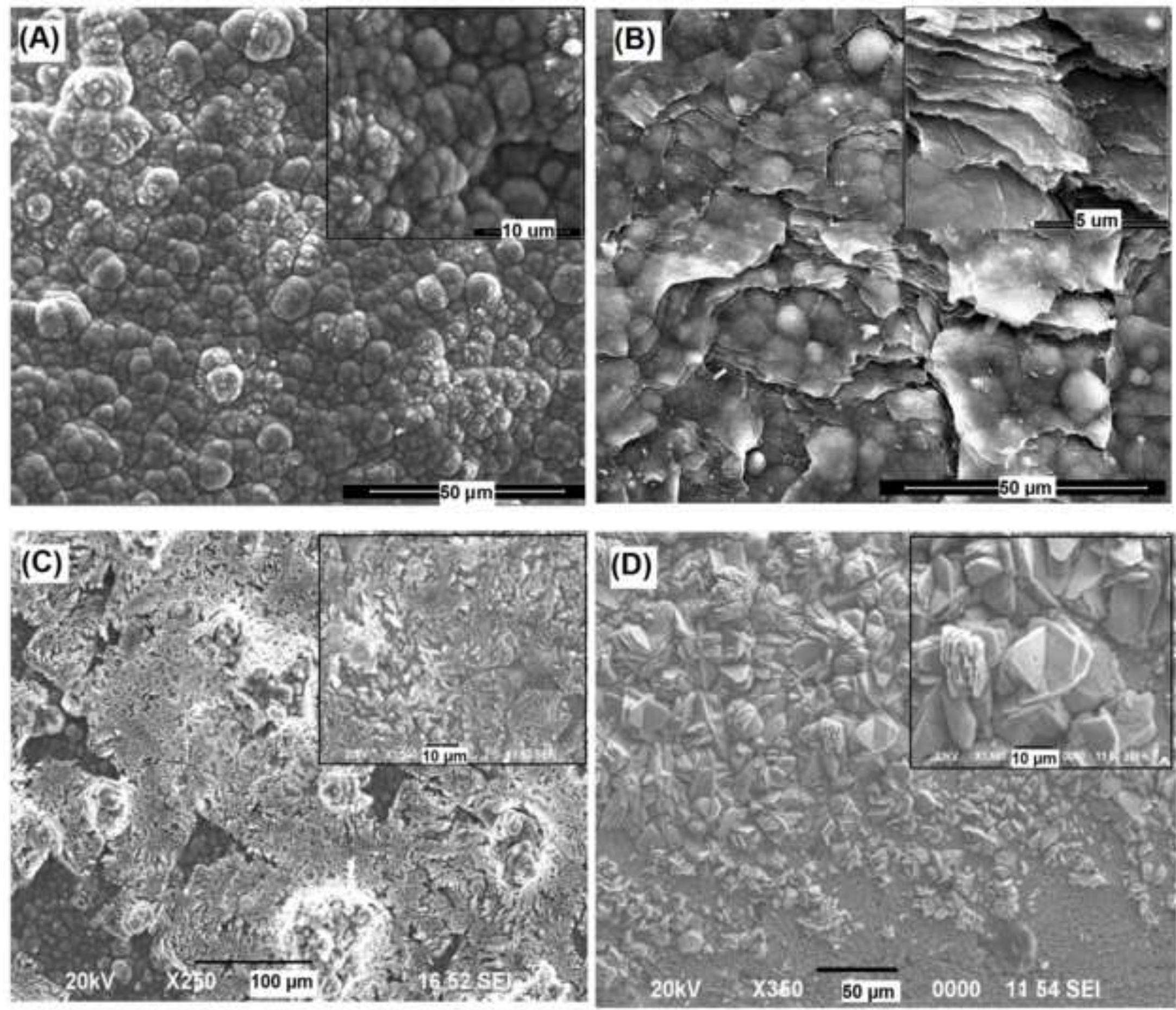


\section{Click here to download Supplementary Material: Supplementary_Information.pdf}

Supplementary Material
Click here to download S 